[0212-7199 (2006) 23: 12; pp 569-572] ANALES DE MEDICINA INTERNA Copyright @ 2006 ARAN EDICIONES, S.L

AN. MED. INTERNA (Madrid) Vol. 23, N. ${ }^{\circ} 12$, pp. 569-572, 2006

\section{Seguimiento de una cohorte de pacientes con gammapatía monoclonal sérica en el Área Sanitaria de Guadalajara}

\author{
M. BATUECAS MOHEDANOํ, F. CARBALLO ÁLVAREZ2,3, L. GARCÍA \\ MENÉNDEZ4.
}

${ }^{1}$ Laboratorio de Análisis Clínicos y ${ }^{2}$ Unidad de Investigación. Hospital Universitario de Guadalajara. ${ }^{3}$ Departamento de Medicina. Universidad de Alcalá. ${ }^{4}$ Laboratorio de Análisis Clínicos y Unidad de Investigación. Hospital El Bierzo. Ponferrada (León)
FOLLOW-UP OF SERUM MONOCLONAL GAMMOPATHY AT GUADALAJARA HEALTH AREA

\section{RESUMEN}

Objetivo: Conocer la evolución clínica de los pacientes del Área Sanitaria de Guadalajara en los que fue diagnosticada una paraproteína sérica.

Material y métodos: Estudio prospectivo de 186 pacientes en los que durante los años 1999 y 2000 se detectó "de novo" una banda monoclonal sérica.

Resultados: La probabilidad acumulada de progresión a enfermedad maligna fue del 4,99\% a los 43 meses en los pacientes cuya paraproteína fue clínicamente soslayada y $2 \%$ a los 23 meses en pacientes diagnosticados de gammapatía monoclonal de significado incierto. La probabilidad acumulada de supervivencia en los pacientes con mieloma múltiple fue $66,7 \%$ a los 21 meses. La tasa condicionada de mortalidad (paciente/mes) a los 4 años debido a enfermedad hematológica fue de $4,48.10^{-4}$ en pacientes clínicamente soslayados, 0 en los diagnosticados de gammapatía monoclonal de significado incierto y $1,388.10^{-2}$ en los diagnosticados de mieloma múltiple.

Discusión: El seguimiento de pacientes con banda monoclonal benigna es fundamental, al menos durante los cuatro primeros años, ya que con ello podría conseguirse una mayor supervivencia en relación con su transformación en maligna.

PALABRAS CLAVE: Gammapatía monoclonal. Paraproteinemias. Discrasia de células plasmáticas. Electroforesis de proteínas séricas. Mieloma múltiple. Macroglobulinemia de Waldenström.

\section{ABSTRACT}

Objective: To study the clinical course of patients with a serum monoclonal protein at Guadalajara Health Area.

Material and methods: Prospective study of 186 patients with a newly diagnosed monoclonal component. They have been collected during the years 1999 and 2000.

Results: The cumulative transformation probability at 43 months was $4.99 \%$ for those patients whose monoclonal gammopathy was overlooked, and 2\% at 23 months for patients with monoclonal gammopathy of undetermined significance. The cumulative probability of survival for patients with multiple myeloma was $66.7 \%$ at 21 months. The conditional mortality rate (patients/months) at 4 years due to haematological disease was $4.48 \times 10^{-4}$ for overlooked patients, 0 for diagnosed of monoclonal gammopathy of undetermined significance and $1.388 \times 10^{-2}$ for multiple myeloma diagnosed.

Discussion: A non malignant M component must be followed up due to it could increase patients' survival rate in relation with transformation in malignant disease.

KEY WORDS: Monoclonal gammopathy. Plasma cells dyscrasias. Electrophoresis. Multiple myeloma. Waldenström macroglobulinemia.

Batuecas Mohedano M, Carballo Álvarez, F, García Menéndez. L. Seguimiento de una cohorte de pacientes con gammapatía monoclonal sérica en el Área Sanitaria de Guadalajara. An Med Interna (Madrid) 2006; 23: 569-572.

\section{INTRODUCCIÓN}

La constante evolución de la técnica analítica utilizada para la detección de bandas monoclonales ha originado un aumento de la sensibilidad del método empleado, y como consecuencia, el descubrimiento de un mayor número de paraproteínas sin que exista sospecha clínica previa de la existencia de una disproteinemia. Por tanto, el hallazgo de una banda monoclonal en el proteinograma sérico es un hecho relativamente frecuente en el laboratorio clínico, ya que gran parte de estas bandas monoclonales detectadas son debidas a la existencia de una gammapatía monoclonal de significado incierto (GMSI) asociada a la edad, que afecta al 3\% de personas mayores de 70 años $(1,2)$. Aún tratándose de una hemopatía benigna estos pacientes deben ser controlados ya que el riesgo de progresión a enfermedad maligna no desaparece, incluso cuando la concentración de componente monoclonal permanezca estable durante años

Trabajo aceptado: 10 de julio de 2006

Correspondencia: Miriam Batuecas Mohedano. C/ Ferrocarril 7, 7º B. Ponferrada. (León). e-mail: miriambatuecas@ hotmail.com. 
(3). A pesar de estas recomendaciones, en un estudio realizado por nuestro grupo de trabajo en el que describimos la frecuencia de presentación del total de las bandas monoclonales detectadas en la provincia de Guadalajara durante dos años, observamos que un porcentaje considerable de las bandas halladas en el laboratorio carecía de un diagnóstico clínico explícito y/o propuesta de seguimiento (4).

El objetivo principal del presente estudio es conocer la evolución a corto plazo de los pacientes de este área sanitaria en los que durante estos dos años fue detectada "de novo" una banda monoclonal sérica. Además se valora el posible beneficio que hubieran obtenido del seguimiento los pacientes cuya banda monoclonal fue clínicamente soslayada.

Otros objetivos son conocer las causas de muerte, si se hubiera producido y los tiempos de supervivencia.

\section{MATERIAL Y MÉTODOS}

Durante los años 1999 y 2000 fueron detectados en el área sanitaria de Guadalajara 222 nuevos casos de gammapatía monoclonal sérica. De ellos, 22 no se estudiaron debido al no acceso a la historia clínica y los 200 restantes fueron clasificados como: no diagnosticados $(119 ; 59,95 \%)$, si en la historia clínica no constaba una estimación diagnóstica explícita o una propuesta de seguimiento; diagnosticados de GMSI (60; $30 \%$; mieloma múltiple (MM) (16; 8\%); enfermedad linfoproliferativa (ELP) $(4 ; 2 \%)$ y plasmocitoma solitario (1; $0,5 \%)$.

Para el estudio prospectivo de estos pacientes no se tuvo acceso al total de la información por lo que se realizó el seguimiento del $93 \%$ de los 200 sujetos que previamente habían sido estudiados. El tiempo medio de seguimiento fue de 33,43 meses ( $\left.\operatorname{IC}_{95 \%}: 31,23 ; 35,63\right)$, siendo mayor en pacientes con GMSI $\left(36,98\right.$ meses) ( IC $\left._{95 \%}: 34,3 ; 39,66\right)$. No se obtuvo información del único paciente diagnosticado de plasmocitoma. Se registraron los datos analíticos y clínicos más relevantes además de la fecha de la última observación, evolución a enfermedad maligna y fecha y causa de fallecimiento si se hubiera producido. Se consideró punto final del seguimiento el fallecimiento del paciente confirmado en la historia clínica y punto final del estudio 4 años. La probabilidad acumulada de progresión a enfermedad maligna fue calculada mediante el método de Kaplan-Meier y la tasa condicionada de mortalidad mediante la fórmula de función de riesgo (actuarial).

\section{RESULTADOS}

\section{MUESTRA DE PARTIDA}

Los 186 pacientes que fueron seguidos tenían una mediana de edad en la fecha del diagnóstico de 76 años (rango: 41-97), el 69,35\% eran mayores de 70 años, ninguno de los pacientes era menor de 40 años en el momento del diagnóstico y el 65,05\% eran hombres. Las medias de inmunoglobulina monoclonal en los pacientes no diagnosticados y diagnosticados de GMSI fueron $6,1 \mathrm{~g} / 1\left(\mathrm{IC}_{95 \%}: 5,17 ; 7,17\right)$ y $6,7 \mathrm{~g} / 1\left(\mathrm{IC}_{95 \%}: 5,3 ; 8,12\right)$ respectivamente, mientras que en pacientes diagnosticados de mieloma fue de $25,8 \mathrm{~g} / 1\left(\mathrm{IC}_{95 \%}\right.$ : $13,79 ; 37,99)$.

\section{SEGUIMIENTO}

Durante el seguimiento se produjo el fallecimiento de 43 $(23,11 \%)$ del total de los pacientes.

En el grupo de los 112 pacientes en los que previamente no se hizo constar un diagnóstico en su historia clínica, clasificados como no diagnosticados, 23 se consideraron perdidos debido a que durante el periodo de seguimiento no consta ningún episodio posterior y fueron, por tanto, 89 los pacientes objeto de estudio. Setenta y ocho de estos pacientes $(76,40 \%)$ continuaban sin diagnóstico o seguimiento de la paraproteína al final del estudio, en 5 (5,62\%) desapareció la banda monoclonal, 4 $(4,49 \%)$ fueron diagnosticados posteriormente de GMSI, 1 $(1,12 \%)$ de Enfermedad de Waldenström (EW) y otro $(1,12 \%)$ de MM. La probabilidad acumulada de progresión a enfermedad maligna en este grupo de pacientes no perdidos fue de 1,3\% a los 8 meses y 4,99\% a los 43 meses (Fig. 1). El único paciente que evolucionó a MM falleció debido a la presencia de la enfermedad hematológica maligna, con un tiempo de supervivencia de 5 días desde que fue diagnosticado; sin embargo los sujetos que fueron diagnosticados de EW y de GMSI continuaban con su proceso al concluir el estudio. La causa de muerte más frecuente de los $29(32,58 \%)$ pacientes que fallecieron debido a enfermedad no relacionada con la presencia de una banda monoclonal (Fig. 2) fue patología neoplásica no hematológica. Durante el periodo de seguimiento de 4 años la tasa condicionada de mortalidad debido a enfermedad no hematológica en este grupo fue de $1,299 \times 10^{-2}$ mientras que las debidas a procesos hematológicos malignos fue de $4,48 \times 10^{-4}$. El tiempo medio de supervivencia de los pacientes que fallecieron por una causa distinta a la presencia de gammapatía monoclonal maligna fue de 18,52 meses $\left(\mathrm{IC}_{95 \%}: 11,70 ; 25,36\right)$.

Respecto a la evolución de los 60 pacientes clasificados durante los años 1999 y 2000 como pacientes con GMSI, en 8 de ellos $(13,33 \%)$ no constaba ningún proceso posterior, por lo que fueron 52 los considerados como no perdidos. Uno $(1,92 \%)$ de estos pacientes evolucionó a MM en un tiempo de 23 meses desde que se diagnosticó la presencia de la banda monoclonal, en $3(5,77 \%)$ individuos desapareció la banda

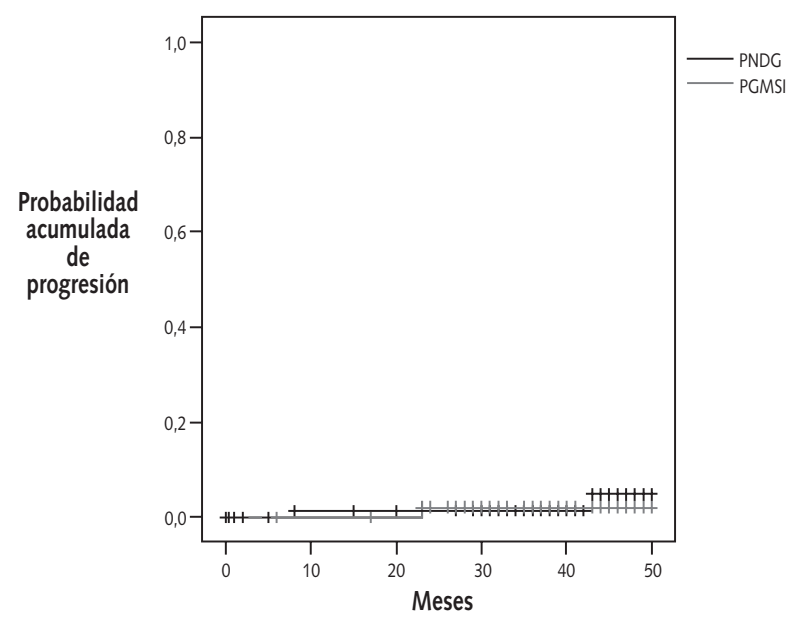

Fig. 1. Probabilidad acumulada de progresión a gammapatía monoclonal maligna en pacientes clasificados como no diagnosticados y diagnosticados de GMSI.

PNDG: Pacientes clasificados como no diagnosticados.

PGMSI: Pacientes diagnosticados de GMSI. 


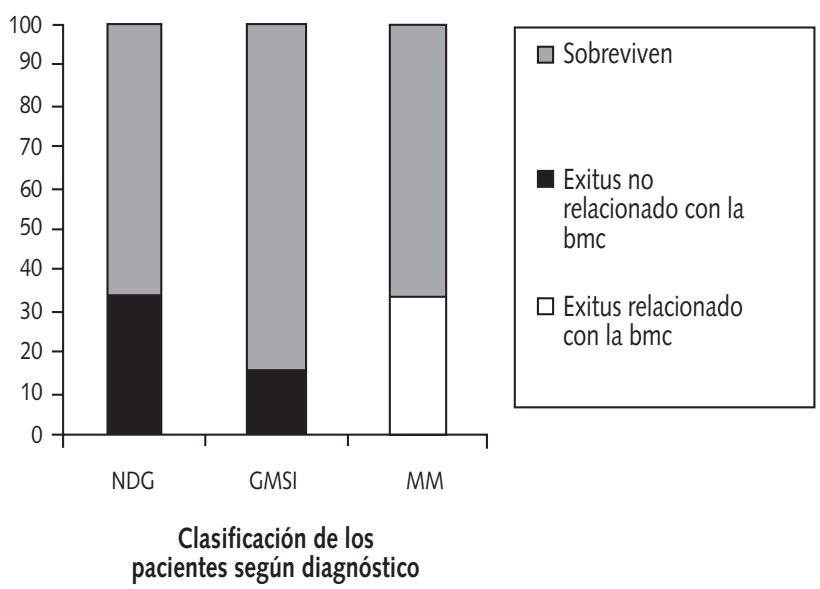

Fig. 2. Evolución de los pacientes en los que se detectó una banda monoclonal sérica.

NDG: Pacientes no diagnosticados.

monoclonal y $48(92,31 \%)$ continuaban estables sin evolución a enfermedad maligna. La probabilidad acumulada de progresión a enfermedad maligna fue de $2 \%$ a los 23 meses (Fig. 1). En este grupo las causas de defunción de los $8(15,38 \%)$ pacientes que fallecieron (Fig. 2) no estaban relacionadas con la presencia de la proteína $\mathrm{M}$ (hemorragia digestiva, neumonía, proceso neoplásico no hematológico, etc.) con un tiempo de supervivencia medio de 24,37 meses ( IC $\left._{95 \%}: 16,26 ; 32,48\right)$. La tasa condicionada de mortalidad debido a causas no hematológicas fue de $5,75 \times 10^{-3}$, mientras que las debidas a procesos hematológicos malignos fue 0 .

En los 12 pacientes diagnosticados de $\mathrm{MM}$ en los que se tuvo acceso a la historia clínica, 6 (50\%) continuaban con su proceso, en $2(16,67 \%)$ constaba una remisión total y 4 (33,33\%) fallecieron como consecuencia de la evolución de su enfermedad (Fig. 2) con un tiempo de supervivencia medio de 250 días (rango: 47-633 días). La probabilidad acumulada de supervivencia fue de $66,7 \%$ a los 21 meses y la tasa condicionada de mortalidad debido a enfermedad hematológica fue $1,388 \times 10^{-2}$ mientras que no hematológica fue 0 .

Respecto a los pacientes con proceso linfoproliferativo, únicamente pudieron revisarse dos de las historias y uno de ellos falleció debido a la progresión del proceso maligno, mientras que el otro continuaba con su patología.

\section{DISCUSIÓN}

A pesar de que la evolución en general de pacientes con gammapatía monoclonal y el riesgo de progresión de hemopatía benigna a maligna han sido muy estudiados, en ninguno de los trabajos revisados se compara la probabilidad de progresión, tasa condicionada de mortalidad y supervivencia de dos grupos de pacientes que, presentando una banda monoclonal, fueron clasificados como no diagnosticados y diagnosticados de GMSI según que su paraproteína fuese clínicamente soslayada o no. Los resultados obtenidos en este trabajo se encuentran limitados debido al no total acceso a las historias clínicas, hecho en el que no hemos podido influir debido a que se trata de un estudio ciego por parte de los clínicos y los pacientes.

En nuestro trabajo la probabilidad acumulada de evolución a enfermedad maligna es similar en el grupo de pacientes cuya gammapatía monoclonal no fue seguida, respecto a los pacientes controlados por la presencia de una GMSI; en cambio, la tasa condicionada de mortalidad debido a enfermedad hematológica maligna es mayor en el grupo de pacientes clasificados como no diagnosticados que en los diagnosticados de GMSI, presentando éstos un tiempo de supervivencia en meses mucho mayor desde que fue diagnosticada la enfermedad maligna.

Debido a que la tasa condicionada de mortalidad se interpreta como una medida de riesgo de mortalidad durante el intervalo de tiempo que ha sido aplicada, se podría deducir que durante los cuatro primeros años tras la detección de la banda monoclonal, el riesgo de fallecer de los pacientes que no presentaron una gammapatía monoclonal maligna en el momento del hallazgo de la banda, es mayor en sujetos que no fueron controlados tras la detección de la paraproteína que en los sujetos que si fueron seguidos. Estos resultados obtenidos no los podemos comparar con los publicados en otras series ya que en la bibliografía revisada no consta un estudio planteado de esta forma.

La frecuencia de bandas monoclonales transitorias obtenidas en nuestro trabajo en los pacientes no diagnosticados y diagnosticados de GMSI ha sido de 5,62 y 5,77\% respectivamente y es similar al 5\% descrita por Kyle et all (3) en pacientes diagnosticados de GMSI, aunque el tiempo de seguimiento de estos autores es mucho mayor que el nuestro. El carácter transitorio de estas bandas podría explicarse en un 50\% de los casos por hepatopatía de carácter viral o tumoral que presentaban los pacientes; en el 50\% restante no hemos conseguido encontrar una explicación lógica para la presencia de esta bandas.

Una proporción alta de los pacientes estudiados fallecieron por causas ajenas a la presencia de la gammapatía monoclonal, siendo este porcentaje mayor en el grupo catalogado como no diagnosticados respecto a los diagnosticados de GMSI, obteniéndose en los no diagnosticados un valor superior al publicado por Giraldo et all (5) para pacientes con GMSI, mientras que para nuestros pacientes con GMSI el valor es inferior al de dichos autores. Como era de esperar, en el grupo de sujetos diagnosticado de MM se produce el mayor porcentaje de fallecimiento debido a la presencia de la hemopatía maligna. Los pacientes clasificados como no diagnosticados y diagnosticados de GMSI presentaron, durante el tiempo que fueron seguidos, una mayor probabilidad de morir por una enfermedad no relacionada con la presencia de gammapatía monoclonal que por la hemopatía maligna, datos coincidentes con los publicados por Kyle et all (3) para pacientes con GMSI.

No hemos podido comparar nuestros datos de supervivencia con otras series debido a la diferencia existente en los tiempos de seguimiento.

Concluimos que el seguimiento de pacientes que presentan una banda monoclonal de carácter benigno es fundamental, al menos durante los primeros cuatro años, ya que con ello podría conseguirse una mayor supervivencia en relación con su transformación en maligna. 


\section{Bibliografía}

1. Axelsson U, Bachman R, Hallen J. Frequency of pathological proteins (M-components) in 6995 sera from an adult population. Acta Med Scand 1996; 179: 235-47.

2. Saleun JP, Vicariot M, Decroff P, Morin JF. Monoclonal gammopathies in the adult population of Finistère, France. J Clin Pathol 1982; 35: 63-8.

3. Kyle RA, Therneau TM, Rajkumar SV, Offord JR, Larson DR, Plevak MF, Melton LJ. A long-term study of prognosis in monoclonal gammo- pathy of undetermined significance. N Engl J Med 2002; 346: 564-9.

4. Batuecas Mohedano M, Piqueras Argüello JA, Carballo Álvarez F, Parra Cid T,Vinssac Gil JL, Granizo Domínguez V. Gammapatía monoclonal: un diagnóstico frecuentemente soslayado. Rev Clin Esp 2005; 205 (5): 207-11.

5. Giraldo P, Rubio-Félix D, Cortés T, et al. Incidencia, características clínico-biológicas y evolutivas de 1203 gammapatías monoclonales (1971-1992). Sangre 1994; 39: 343-50. 